\title{
DINÂMICA DA MATA ATLÂNTICA 510 ANOS APÓS O DESCOBRIMENTO: TENDENCIAS E DRIVERS ATUAIS
}

\author{
Carolina Marques Guilen Lima \\ Ricardo Alexandrino Garcia*
}

\section{Resumo}

Apesar de ser um bioma em situação crítica, pouco se conhece da atual dinâmica da Mata Atlântica e dos fatores que a influenciam. Este trabalho apresenta as principais mudanças na cobertura vegetal do bioma entre 1995 e 2006, relacionando-as com informações de uso do solo, população e economia em nível municipal, por meio de regressão linear pelo método stepwise. Múltiplas escalas foram avaliadas: bioma, macrorregiões e estados. Os resultados mostraram que o saldo global ainda é de decremento da área remanescente do bioma. Por outro lado, observou-se um padrão de transição florestal em aproximadamente $60 \%$ dos municípios. Tal fenômeno está provavelmente associado à legislação ambiental e à fiscalização por parte dos órgãos responsáveis. Inserção de novas variáveis e avaliação da autocorrelação espacial de serão realizados para aprofundamento da pesquisa sobre este tema.

Palavras chave:Mata Atlântica, desmatamento, transição florestal, fatores socioeconômicos.

\begin{abstract}
Despite its critical situation, little is known about the present dynamics of Atlantic Forest and the drivers by which it is influenced. This paper presents the main changes in land cover on the biome domain between 1995 and 2006, relating such changes to information on land use, population and economy, at the municipal level. The method used is stepwise linear regression. Multiple scales were analyzed: biome, macro regions and states. Results shows that the global balance is decrement of the biome remnant area. On the other hand, a pattern of forest transition was observed on approximately $60 \%$ of the municipalities. Such phenomenon is probably associated to environmental legislation and control of the authoritative institutions. Use of new variables and evaluation of spatial autocorrelation will be done to deepen the research on this subject.
\end{abstract}

Keywords: Atlantic Forest, deforestation, forest transition, LUCC models, socioeconomic drivers. INTRODUÇÃO

\footnotetext{
- Centro de Sensoriamento Remoto, Universidade Federal de Minas Gerais, carolmg@gmail.com.

*Laboratório de Estudos Territoriais, Universidade Federal de Minas Gerais, Dr., alexandrinogarcia@gmail.com.
} 
Possivelmente os maiores impactos antrópicos sobre o meio ambiente estão relacionados à mudança na cobertura da terra. Estradas são abertas, áreas naturais convertidas em cidades e extensas áreas de florestas são substituídas por lavouras e pastagens, entre outras intervenções.

No Brasil, país de dimensões continentais e detentor de porção significativa da biodiversidade mundial, mudanças do uso da terra têm ocorrido desde a formação das sociedades indígenas ancestrais, porém a escala das intervenções teve um aumento significativo a partir da chegada dos colonizadores portugueses. A história da ocupação e utilização do solo no Brasil ocorreu de forma heterogênea em cada porção do seu território. A Amazônia, bioma brasileiro mais estudado, teve um histórico de ocupação relativamente recente, sendo que de 1960 a 1980 o desmatamento foi principalmente motivado por forças políticas. De 1990 em diante, forças de mercado passaram a ter um papel preponderante na região (SILVA 2009). Já a Mata Atlântica teve sua ocupação e seu desenvolvimento econômico iniciados logo no início da história de colonização do Brasil. Com a extração de pau-brasil, a exploração de minério e a rápida expansão das culturas de café e cana-de-açúcar, as terras do sudeste brasileiro foram rapidamente ocupadas por povoados e cidades, às custas de um intensivo desmatamento e fragmentação do bioma. Ao longo dos séculos, a degradação do bioma estendeu-se para o Sul, Centro-Oeste e mais recentemente ao Nordeste de seu domínio. Hoje, além da pressão de expansão agropecuária, a Mata Atlântica é ameaçada pela expansão urbana, devido ao denso povoamento da costa litorânea e principalmente da região sudeste, onde é comum o fenômeno de expansão de grandes metrópoles sobre áreas rurais, inicialmente lotes para lazer e residências mais afastadas no centro urbano. Apesar de a história de ocupação do bioma ser de domínio comum, pouco tem sido cientificamente investigado sobre a atual dinâmica dos remanescentes do bioma e sobre as forças que a determinam.

$\mathrm{Na}$ busca de compreender as variações de uso e ocupação do solo e suas possíveis consequências sobre os recursos naturais e a biota, a elaboração de modelos tem se mostrado bastante útil, proporcionando um recorte e uma simplificação do sistema natural e permitindo, assim, seu melhor entendimento, além de simulações com projeções de possíveis futuros com variados cenários. Diversas são as possibilidade de modelagem ambiental, desde formulações matemáticas simples a modelos econométricos e modelos espacialmente explícitos,envolvendo vários graus de complexidade, e podendo ou não incorporar feedbacks, dimensões temporais e espaciais.

No Brasil, os modelos que buscam entender a dinâmica de mudança de cobertura e uso do solo concentram-se claramente no bioma amazônico (LORENA \& LAMBIN 2009; MICHALSKI et al. 2010; SILVA 2009; SOARES-FILHO et al. 2008; REIS et al. 1997; AGUIAR 
et al. 2007; ANDERSEN 1997; PERZ \& SKOLE 2003; dentre outros). A Mata Atlântica, por sua vez, é menos estudada, embora se encontre em situação extremamente crítica, restando apenas cerca de $12 \%$ de sua área original remanescente na forma de fragmentos em sua maioria pequenos e dispersos no território brasileiro (RIBEIRO et al. 2009). Ao contrário do que ocorre para a Amazônia, estudos que buscam investigar a dinâmica atual da Mata Atlântica são relativamente escassos (RIBEIRO et al. 2009; TEIXEIRA et al. 2009), e menor ainda é o volume de estudos que investigam as causas socioeconômicas de tal dinâmica (BAPTISTA \& RUDEL 2006; EHLERS 2003).

Uma vez que modelos de regressão linear são ferramentas bastante úteis na exploração de possíveis drivers para os processos de desmatamento e regeneração florestais, propõe-se neste estudo utilizar esses métodos para explorar quais variáveis socioeconômicas influenciam hoje a dinâmica da Mata Atlântica.

\section{MÉTODOS}

A área de estudo corresponde ao domínio geográfico da Mata Atlântica em toda sua extensão, de acordo com o Decreto Federal 6.660/2008, que regulamenta os limites do bioma (Figura 1).

Buscou-se uma abordagem multiescalar, conforme recomendam Wu \&Hobbs (2002). Nesse sentido, o estudo foi elaborado nas seguintes escalas: domínio completo do bioma, por macrorregião geográfica, por estado. Para o último nível de abordagem, foram selecionados os estados da região Sudeste, por ser de maior conhecimento dos autores, permitindo uma análise inicial mais acurada. Também foram analisados os estados da Bahia e do mato Grosso do Sul, por terem apresentado padrões extremos de regeneração e desmatamento. 


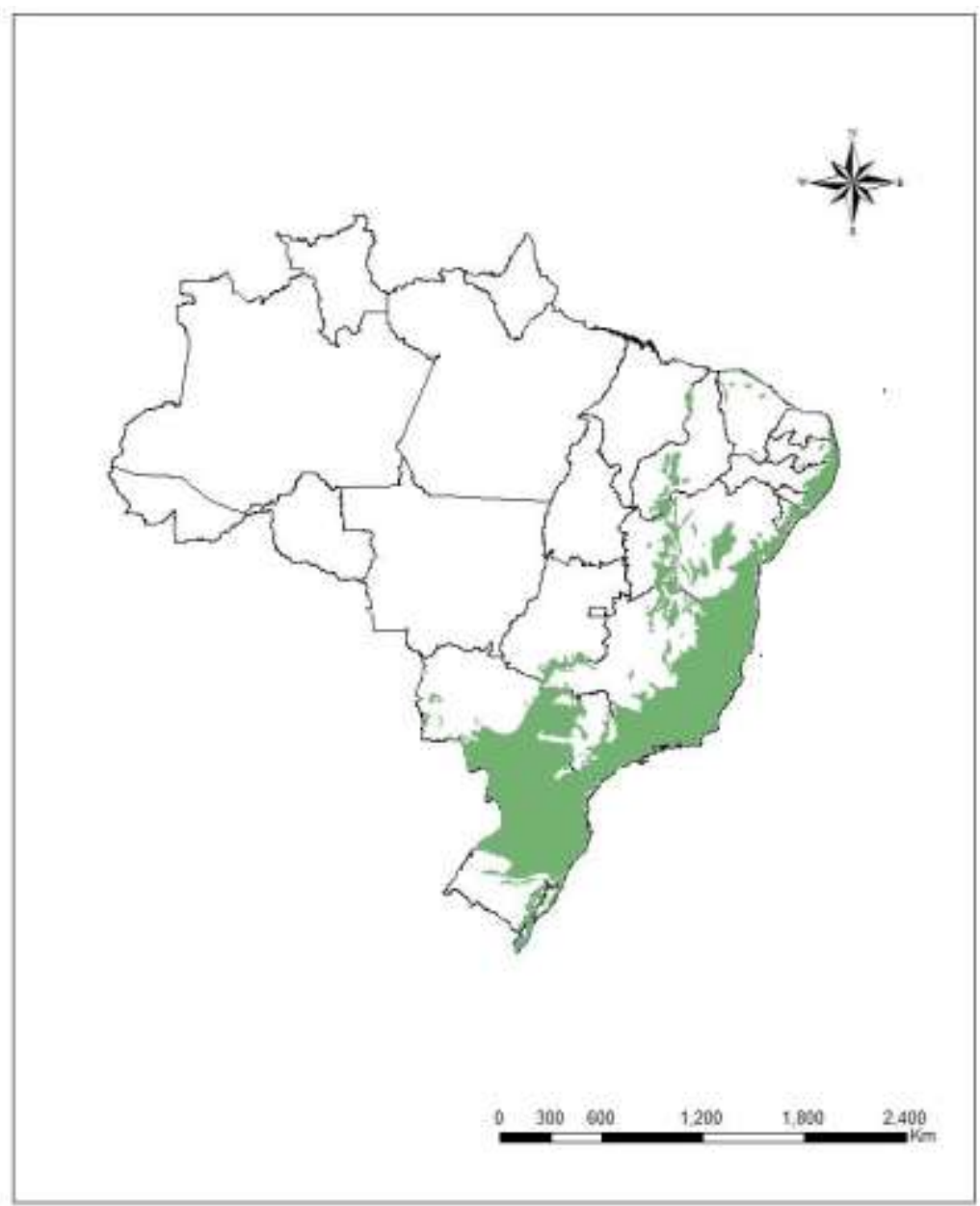

Figura 1 - Mapa da extensão do domínio geográfico do bioma Mata Atlântica, de acordo com o Decreto Federal 6.660/2008.

As variáveis dependentes e independentes foram compiladas para o período entre 1995 e 2006, a partir de dados do Instituto Brasileiro de Geografia e Estatística (IBGE) e do Instituto de Pesquisa Econômica Aplicada (IPEA), obtidos por meio das plataformas de pesquisa Sistema IBGE de Recuperação Automática - SIDRA (http://www.sidra.ibge.gov.br/bda/tabela/ listabl.asp?c $=264 \& z=t \& o=11) \quad$ e IPEADATA (http://www.ipeadata.gov.br $/$ ipeaweb.dll/ ipeadata? Tick=361793593).

Foram selecionados os seguintes dados do Censo Agropecuário do IBGE: área de mata nativa, área de mata legalmente protegida, área de lavoura, área de mata plantada, área de pastagem e escolaridade do produtor. Do Censo Demográfico do IBGE foram utilizados dados de população urbana e rural.

Em relação aos índices econômicos, foram utilizados dados do IPEA (PIB) e índices de industrialização, terceirização, dinamismo municipal e dinamismo migratóriocalculados por um dos autores. Os índices de industrialização (IDC) e de terceirização (ITC) foram elaborados segundo Garcia \& Lemos (2009), e suas fórmulas são apresentadas na Tabela 1. O índice de Cadernos do Leste 
dinamismo municipal (DIMUN) refere-se à quantidade de municípios que enviam pessoas para estudar ou trabalhar no município alvo da análise, e o índice de dinamismo de imigração (DIMIG) refere-se à quantidade de municípios que enviou pessoas para residir no município alvo. Ambos indicadores foram obtidos com base nos microdados do Censo Demográfico de 2000.

As áreas municipais foram obtidas por meio de cálculo realizado em plataforma de geoprocessamento (ArcGIS). Para evitar que municípios muito grandes, com consequentes extensos desmatamentos e regenerações distorcessem a avaliação, todos os dados foram relativizados, sendo divididos pela área municipal. Dessa forma, os dados de uso e ocupação do solo utilizados representam a porcentagem daquela cobertura em relação à extensão do município, a população urbana e rural é apresentada em termos de densidade populacional etc.

Dos dados de uso e ocupação do solo, bem como os de PIB e população urbana e rural, foram extraídos os deltas temporais (At1-At0, exceto para desmatamento, em que o delta = Dt0Dt1). Todos os valores nulos e zero foram inicialmente excluídos, para evitar que a subtração com um dos fatores ausentes gerasse artefatos de deltas irreais. Os deltas foram utilizados como variáveis dependente e independentes, conforme apresentado na Tabela 1.

\begin{tabular}{|l|l|}
\hline \multicolumn{2}{|c|}{ Variável dependente } \\
\hline DESMT & $\begin{array}{l}\text { Porcentagem de estabelecimentos rurais do município coberta por mata nativa em } \\
1995, \text { menos porcentagem de mata nativa remanescente em 2006. }\end{array}$ \\
\hline \multicolumn{2}{|c|}{ Variáveis independentes } \\
\hline AMPSILV & $\begin{array}{l}\text { Porcentagem de estabelecimentos rurais do município coberta por silvicultura em } \\
2006, \text { menos porcentagem de silvicultura em 1995. }\end{array}$ \\
\hline AMPPTPLA & $\begin{array}{l}\text { Porcentagem de estabelecimentos rurais do município coberta por pastagens } \\
\text { plantadas em 2006, menos porcentagem de pastagens plantadas em 1995. }\end{array}$ \\
\hline AMPLAV & $\begin{array}{l}\text { Porcentagem de estabelecimentos rurais do município coberta por lavoura em } \\
2006, \text { menos porcentagem de lavoura em 1995. }\end{array}$ \\
\hline APPRL & $\begin{array}{l}\text { Porcentagem de estabelecimentos rurais do município coberta por matas nativas } \\
\text { legalmente protegidas (áreas de preservação permanente e reservas legais) em } \\
\text { 2006, menos porcentagem de matas legalmente protegidas em 1995. }\end{array}$ \\
\hline
\end{tabular}




\begin{tabular}{|l|l|}
\hline ENSSUP & $\begin{array}{l}\text { Quantidade de produtores agrícolas que completaram o ensino superior, de } \\
\text { acordo com Censo Agropecuário de 2006. }\end{array}$ \\
\hline AMPOPRU & $\begin{array}{l}\text { Densidade de população residente na área rural em } 2007 \text { menos densidade de } \\
\text { população residente na área rural em 1996. }\end{array}$ \\
\hline AMPOPURB & $\begin{array}{l}\text { Densidade de população residente na área urbana em } 2007 \text { menos densidade de } \\
\text { população residente na área urbana em } 1996 .\end{array}$ \\
\hline PIB06 & $\begin{array}{l}\text { Produto Interno Bruto (PIB) do município em } 2006 \text { (R\$ de 2000(mil)), dividido } \\
\text { pela área municipal. }\end{array}$ \\
\hline AMPIB & $\begin{array}{l}\text { PIB do município em 2006 menos PIB do município em 2003, dividido pela área } \\
\text { municipal. }\end{array}$ \\
\hline
\end{tabular}




\begin{tabular}{|c|c|}
\hline DIMIG & $\begin{array}{l}\text { Quantidade de municípios que enviaram pessoas para residir no município alvo, } \\
\text { no período de 01/08/1995 e 01/08/2000. }\end{array}$ \\
\hline DIMUN & $\begin{array}{l}\text { Quantidade de municípios que enviaram pessoas para estudar ou trabalhar no } \\
\text { município alvo, no período de 01/08/1995 e 01/08/2000. }\end{array}$ \\
\hline ITC & $\begin{array}{l}\qquad I T_{j}=\begin{array}{l}\mathrm{a} \cdot \mathrm{v}_{\cdot \mathrm{j}} \\
\left(\mathrm{PIB}_{\mathrm{j}}\right)\end{array} \\
\text { Índice de Terciarização em 2000; onde: a.v.sj é o v.a. do setor Serviços na região } \\
\mathrm{j} \text { e PIBj é o produto interno bruto da região j. }\end{array}$ \\
\hline IDC & $\begin{array}{l}\qquad I D_{j}=\begin{array}{l}\mathrm{a} \cdot \mathrm{v}_{\bullet \mathrm{j}} \\
\left(\mathrm{PIB}_{\mathrm{j}}\right)\end{array} \\
\text { Índice de Industrialização em 2000; onde: a.v.sj é o v.a. do setor Industrial na } \\
\text { região j e PIBj é o produto interno bruto da região j. }\end{array}$ \\
\hline
\end{tabular}

A opção de se quantificar o desmatamento a partir dos dados do Censo Agropecuário teve por objetivo utilizar a mesma fonte das variáveis independentes (IBGE), minimizando assim possíveis divergências devido a diferentes metodologias de obtenção dedados. Optou-se por não se utilizar os mapas do SOS Mata Atlântica/INPE, fonte frequentemente utilizada para estudos do bioma, porque tais mapeamentos têm tido variaçãosignificativa na metodologia a cada edição do Atlas, de maneira que a utilização desses dadosem série temporal fica limitada e os erros associados são incertos. Reconhece-se, por outro lado, que os dados do Censo Agropecuário também estão sujeitos a erros. Os dados aqui utilizados são fundamentados em entrevistas, ou seja, os valores utilizados para mata nativa epara as demais tipologias de uso do solo são aqueles declarados pelos produtores rurais.

Como a elaboração de modelos para a dinâmica da Mata Atlântica é ainda incipiente, propôs-se a abordagem baseada em dados como a mais adequada. Através dessa abordagem se constrói, com base em tratamentos estatísticos, um modelo que melhor se ajuste aos dados utilizados como input na análise. Essa estratégia é útil quando as relações entre as variáveis ainda não estão claras para a ciência, ficando, portanto, inviável o uso de um modelo baseado em conhecimento.

Considerando a quantidade de remanescentes (área em hectares) como variável dependente e as variáveis socioeconômicas e de uso do solo como variáveis independentes e potencialmente explicativas, foi realizado procedimento para seleção e avaliação das variáveis mais explicativas por meio de regressão logística stepwise, com auxílio do software IBM SPSS Statistics. O procedimento stepwise executa automaticamente vários modelos de regressão com diferentes combinações das variáveis explicativas, adicionando ou removendo variáveis 
independentes em cada iteração. Dessa forma, o método permite a seleção de um subconjunto de variáveis preditoras com maior capacidade de explicação da variável independente, além de fornecer índices do grau de ajuste dos modelos experimentados e o valor de coeficiente e a significância associada a cada variável explanatória.

É importante ressaltar que, pela natureza dos dados utilizados, foi estudada a dinâmica dos remanescentes de Mata Atlântica situados dentro de propriedades consideradas como estabelecimentos rurais pelo IBGE nos Censos Agropecuários de 1995 e 2006. Dessa forma, não foram aqui abordadas informações relativas a fragmentos florestais situados em terras públicas e outros tipos de propriedade de solo.

\section{RESULTADOS}

Um total de 3.411 municípios foi analisado, correspondendo ao domínio geográfico da Mata Atlântica. Desses, 3.086 corresponderam a casos válidos para a análise. Desse conjunto, segundo os dados do Censo Agropecuário, 39,79\% correspondem a municípios que tiveram desmatamento líquido no período analisado (1995 a 2006), e 60,21\% correspondem a municípios que tiveram regeneração líquida, ou seja, cuja quantidade de mata nativa declarada em 2006 excedeu a quantidade informada para 1995. Apesar de mais municípios terem tido regeneração que desmate, em termos de área absoluta continua havendo perda de vegetação nativa: os dados indicam que 13,98 milhões de hectares foram desmatados e 11,68 milhões de hectares regenerados no período analisado (1995 a 2006), resultando num

saldo de aproximadamente 2,3 milhões de hectares de Mata Atlântica perdidos nesses 11 anos.

Em relação à distribuição espacial dessa dinâmica, observou-se que todas as regiões apresentaram mais municípios em processo de regeneração que de desmate, exceto a região Nordeste (Figura 2). Já em termos de área líquida desmatada x regenerada, o Centro-Oeste destaca-se como região desmatadora e o Nordeste como região regeneradora do bioma (Figura 3). A tendência dessas duas regiões é impulsionada pelo padrão extremamente marcante que foi identificado nos estados, respectivamente, do Mato Grosso do Sul e Bahia (Figura 4). 


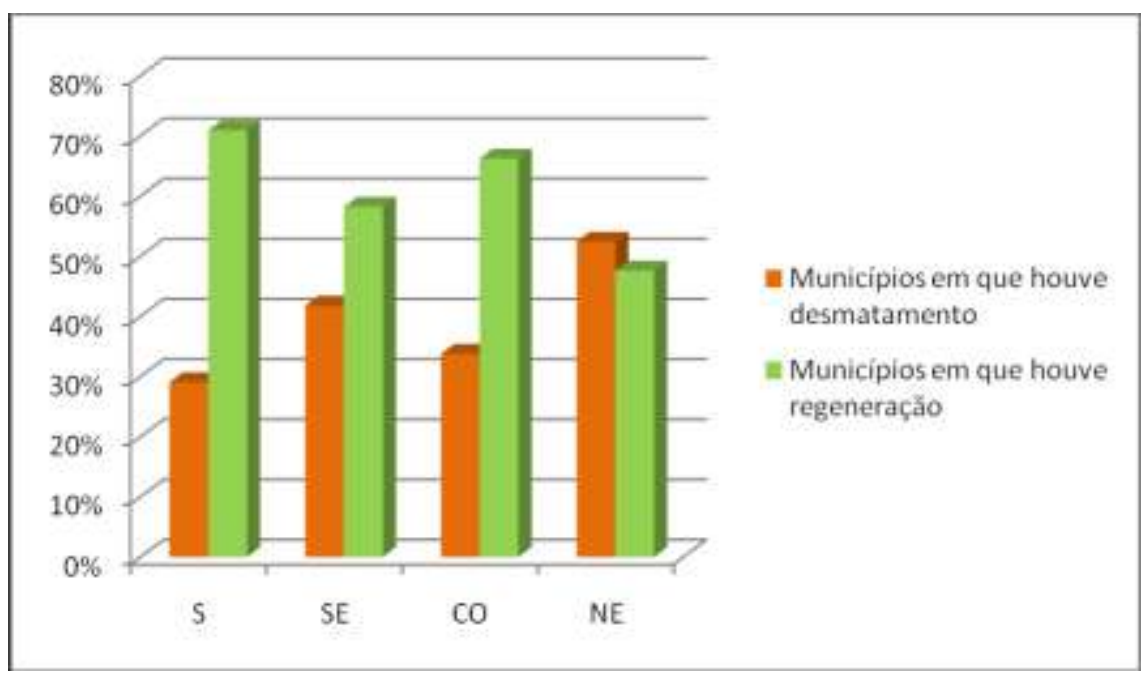

Figura 2 - Porcentagem de municípios de cada região em que houve desmatamento/ regeneração de Mata Atlântica entre 1995 e 2006.

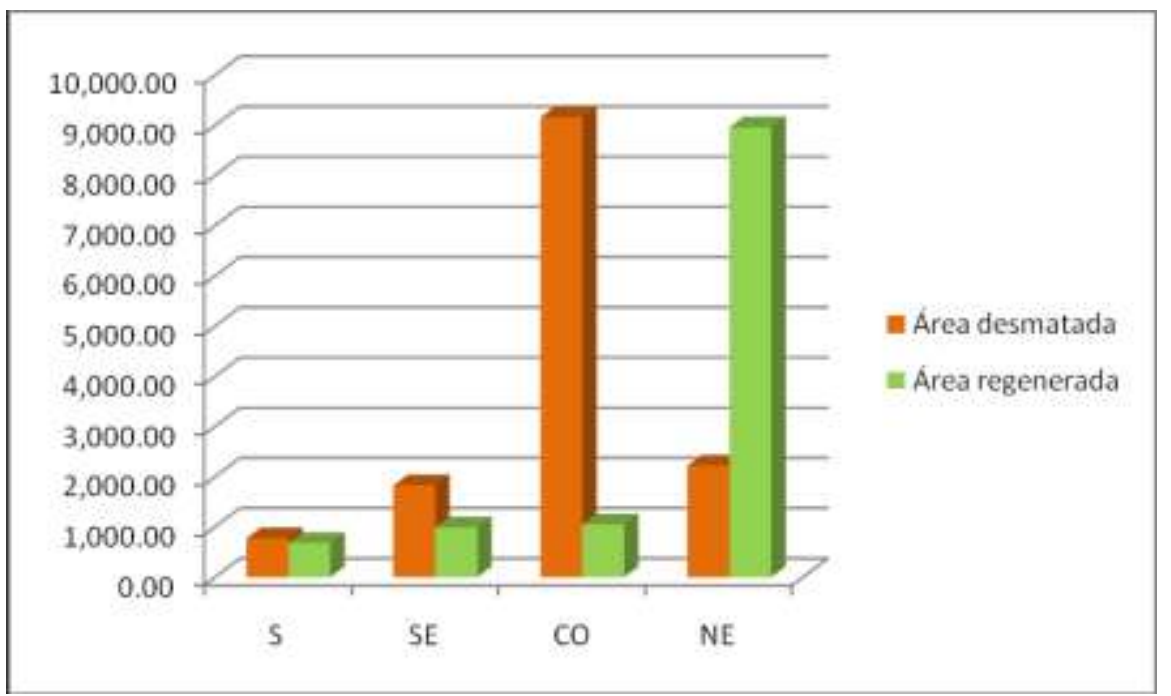

Figura 3 - Quantidade de área de Mata Atlântica desmatada/regenerada por região, no período de 1995 a 2006 (em 1.000ha). 


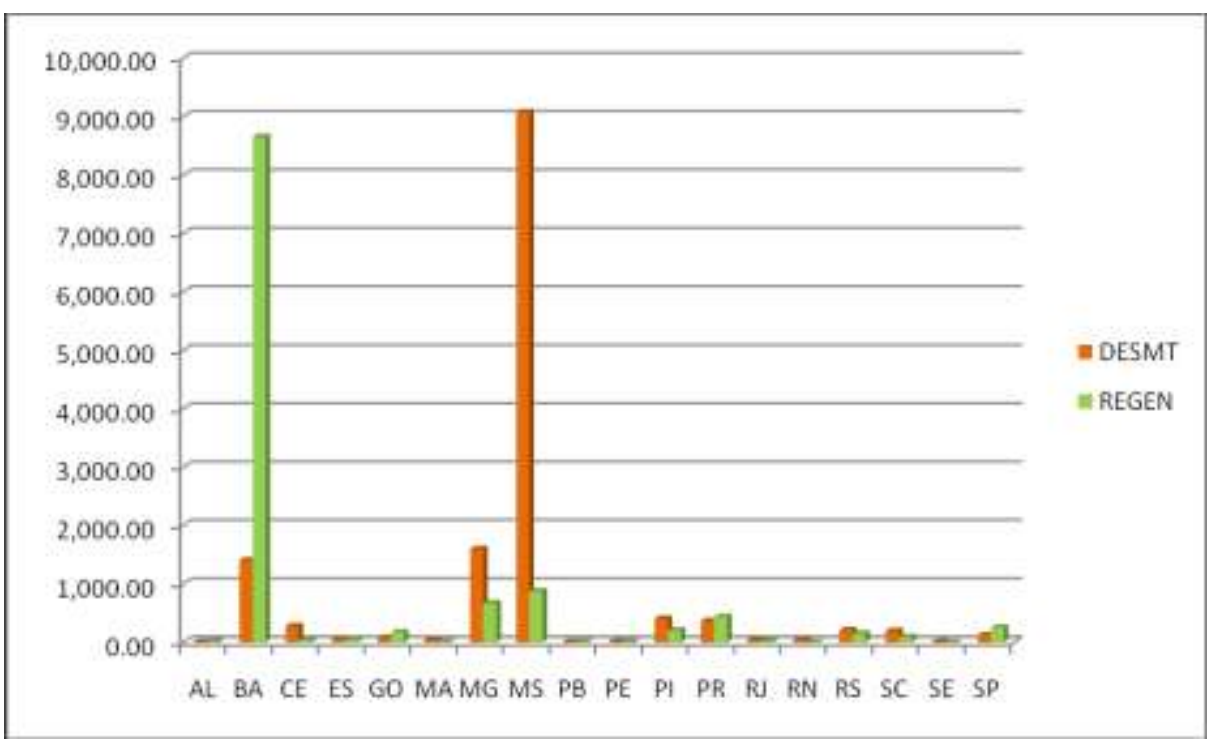

Figura 4 - Quantidade de Mata Atlântica desmatada/regenerada em cada Estado, no período de 1995 a 2006 (em 1.000ha).

Na Tabela 2 são apresentados os resultados da regressão stepwise completa, para o bioma como um todo e englobando todos os municípios do domínio da Mata Atlântica com dados válidos. Após essa análise geral, para refinar as regressões e captar tendências diferenciadas, o total de municípios foi separado em dois subconjuntos: o dos municípios que regeneram mais do que desmatam (daqui em diante chamados, para fins de simplificação, "municípios regeneradores") e o grupo dos que desmataram mais do que regeneraram no período ("municípios desmatadores"). Os subconjuntos foram então submetidos ao stepwise, e os resultados dessas análises são apresentados na Tabela 3. 


\begin{tabular}{|c|c|c|c|c|c|c|}
\hline \multicolumn{7}{|c|}{ Model Summary } \\
\hline Model & $\mathbf{R}$ & R Square & Adjusted $\mathrm{R}^{2}$ & \multicolumn{3}{|c|}{ Std. Error of the Estimate } \\
\hline \multicolumn{7}{|c|}{$\begin{array}{l}\text { a. Predictors: (Constant), AMPIBind_A, AMPSILV_A, PIB_IND, DIMUN, AMPOPRU_A, AMPOPURB_A, DIMIG, AMPLAV_A, } \\
\text { APPRLO6_A, AMPPTPLA_A, IDC, AREA, ENSSUP, ITC, AMPIB_A }\end{array}$} \\
\hline \multicolumn{7}{|c|}{ Coefficients $^{a}$} \\
\hline \multirow{2}{*}{\multicolumn{2}{|c|}{ Model }} & \multicolumn{2}{|c|}{$\begin{array}{l}\text { Unstandardized } \\
\text { Coefficients }\end{array}$} & $\begin{array}{l}\text { Standardized } \\
\text { Coefficients }\end{array}$ & $\mathbf{t}$ & Sig. \\
\hline & & B & Std. Error & Beta & & \\
\hline \multirow{16}{*}{1} & (Constant) & $-0,006$ & 0,004 & & $-1,428$ & 0,154 \\
\hline & AREA & 4,031E-7 & 0,000 & 0,490 & 16,751 & 0,000 \\
\hline & AMPSILV & $-0,847$ & 0,071 & $-0,216$ & $-11,965$ & 0,000 \\
\hline & AMPPTPLA & $-0,230$ & 0,022 & $-0,210$ & $-10,443$ & 0,000 \\
\hline & AMPLAV & $-0,102$ & 0,032 & $-0,063$ & $-3,172$ & 0,002 \\
\hline & APPRL & $-1,221$ & 0,043 & $-0,864$ & $-28,637$ & 0,000 \\
\hline & ENSSUP & $-3,300 \mathrm{E}-7$ & 0,000 & $-0,064$ & $-2,157$ & 0,031 \\
\hline & AMPOPRU & $-0,001$ & 0,011 & $-0,002$ & $-0,093$ & 0,926 \\
\hline & AMPOPURB & $-0,001$ & 0,004 & $-0,003$ & $-0,173$ & 0,863 \\
\hline & ITC & 0,057 & 0,054 & 0,033 & 1,052 & 0,293 \\
\hline & IDC & 0,033 & 0,078 & 0,011 & 0,418 & 0,676 \\
\hline & DIMIG & 0,268 & 0,334 & 0,014 & 0,803 & 0,422 \\
\hline & DIMUN & $-3,594 \mathrm{E}-5$ & 0,000 & $-0,032$ & $-0,890$ & 0,374 \\
\hline & AMPIB & $-5,234 \mathrm{E}-5$ & 0,000 & $-0,020$ & $-0,597$ & 0,550 \\
\hline & PIB_IND & $4,743 \mathrm{E}-13$ & 0,000 & 0,015 & 0,854 & 0,393 \\
\hline & AMPIBind & $-1,422 \mathrm{E}-12$ & 0,000 & $-0,013$ & $-0,713$ & 0,476 \\
\hline
\end{tabular}

Tabela 2 - Resultados da regressão stepwise para o bioma da Mata Atlântica. 
Tabela 3 - Graus de ajuste dos modelos e variáveis selecionadas pelo método stepwise para cada subconjunto de municípios e escalas analisados.

\begin{tabular}{|c|c|c|c|c|c|c|c|c|c|c|}
\hline \multicolumn{11}{|c|}{$\begin{array}{l}\text { DESMATAM } \\
\text { Variável dependente: proporção desmatada no período }\end{array}$} \\
\hline & \multicolumn{2}{|c|}{ BIOMA } & \multicolumn{2}{|c|}{$\mathbf{s}$} & \multicolumn{2}{|c|}{ SE } & \multicolumn{2}{|c|}{ NE } & \multicolumn{2}{|c|}{ co } \\
\hline Ajuste melhor Modelo (R) & \multicolumn{2}{|c|}{0,631} & \multicolumn{2}{|c|}{0,652} & \multicolumn{2}{|c|}{0,702} & \multicolumn{2}{|c|}{0,788} & \multicolumn{2}{|c|}{0,752} \\
\hline $\begin{array}{l}\text { (+) Variáveis selecionadas } \\
\text { e coeficientes normalizados }\end{array}$ & AREA & 0,708 & $\begin{array}{l}\text { APPRL } \\
\text { IDC }\end{array}$ & $\begin{array}{l}0,587 \\
0,144\end{array}$ & AREA PIB & $\begin{array}{l}0,756 \\
0,117\end{array}$ & AREA & 1,052 & & \\
\hline $\begin{array}{l}\text { (-) Variáveis selecionadas } \\
\text { e coeficientes normalizados }\end{array}$ & $\begin{array}{c}\text { APPRL } \\
\text { AMPSILV } \\
\text { AMPLAV } \\
\text { ENSSUP }\end{array}$ & $\begin{array}{l}-0,181 \\
-0,177 \\
-0,092 \\
-0,077\end{array}$ & AMPSILV & $-0,132$ & $\begin{array}{l}\text { AMPSILV } \\
\text { APPRL } \\
\text { IDC } \\
\text { AMPLAV } \\
\text { DIMUN }\end{array}$ & $\begin{array}{l}-0,183 \\
-0,182 \\
-0,158 \\
-0,135 \\
-0,117\end{array}$ & $\begin{array}{l}\text { APPRL } \\
\text { DIMUN }\end{array}$ & $\begin{array}{l}-0,283 \\
-0,201\end{array}$ & ENSSUP & $-0,752$ \\
\hline \multicolumn{11}{|c|}{$\begin{array}{l}\text { REGENERAM } \\
\text { variável dependente: proporção regenerada no periodo }\end{array}$} \\
\hline & \multicolumn{2}{|c|}{ BIOMA } & \multicolumn{2}{|c|}{$\mathbf{s}$} & \multicolumn{2}{|c|}{ SE } & \multicolumn{2}{|c|}{ NE } & \multicolumn{2}{|c|}{ CO } \\
\hline Ajuste melhor Modelo (R) & \multicolumn{2}{|c|}{\begin{tabular}{|r|}
0,829 \\
\end{tabular}} & \multicolumn{2}{|c|}{0.781} & \multicolumn{2}{|c|}{0.722} & \multicolumn{2}{|c|}{0.979} & \multicolumn{2}{|c|}{0.671} \\
\hline $\begin{array}{l}\text { (+) Variáveis selecionadas } \\
\text { e coeficientes normalizados }\end{array}$ & $\begin{array}{c}\text { APPRL } \\
\text { AMPPTPLA } \\
\text { AMPSILV }\end{array}$ & $\begin{array}{l}0,996 \\
0,239 \\
0,106\end{array}$ & $\begin{array}{l}\text { APPRL } \\
\text { AMPSILV } \\
\text { IDC } \\
\text { AMPLAV }\end{array}$ & $\begin{array}{l}0,775 \\
0,117 \\
0,101 \\
0,077\end{array}$ & $\begin{array}{c}\text { APPRL } \\
\text { AMPPTPLA } \\
\text { AMPLAV } \\
\text { DIMIG }\end{array}$ & $\begin{array}{l}0,822 \\
0,179 \\
0,128 \\
0,070\end{array}$ & $\begin{array}{l}\text { APPRL } \\
\text { AMPSILV }\end{array}$ & $\begin{array}{l}1,026 \\
0,079\end{array}$ & $\begin{array}{l}\text { APPRL } \\
\text { AMPSILV }\end{array}$ & $\begin{array}{l}0,455 \\
0,356\end{array}$ \\
\hline $\begin{array}{l}\text { (-) Variáveis selecionadas } \\
\text { e coeficientes normalizados }\end{array}$ & $\begin{array}{l}\text { AREA } \\
\text { ENSSUP }\end{array}$ & $\begin{array}{l}-0,141 \\
-0,092\end{array}$ & AREA & $-0,114$ & $\begin{array}{l}\text { AREA } \\
\text { AMPSILV }\end{array}$ & $\begin{array}{l}-0,144 \\
-0,114\end{array}$ & AREA & $-0,147$ & & \\
\hline
\end{tabular}

...continuação

\begin{tabular}{|c|c|c|c|c|c|c|c|c|c|c|}
\hline \multicolumn{11}{|c|}{$\begin{array}{l}\text { DESMATAM } \\
\text { Variável dependente: proporção desmatada no periodo }\end{array}$} \\
\hline & \multicolumn{2}{|l|}{ ES } & \multicolumn{2}{|l|}{ MG } & \multicolumn{2}{|l|}{$\mathbf{R J}$} & \multicolumn{2}{|l|}{ SP } & \multicolumn{2}{|c|}{ MS } \\
\hline Ajuste melhor Modelo (R) & \multicolumn{2}{|c|}{0,774} & \multicolumn{2}{|c|}{0,739} & \multicolumn{2}{|c|}{0,796} & \multicolumn{2}{|c|}{0,823} & \multicolumn{2}{|c|}{0,779} \\
\hline $\begin{array}{l}\text { (t) Variáveis selecionadas } \\
\text { e coeficientes normalizados }\end{array}$ & AREA & 0,774 & AREA & 1,020 & APPRL & 0,551 & $\begin{array}{c}\text { APPRL } \\
\text { AMPSILV }\end{array}$ & $\begin{array}{l}0,896 \\
0,233\end{array}$ & & \\
\hline $\begin{array}{l}\text { (-) Variáveis selecionadas } \\
\text { e coeficientes normalizados }\end{array}$ & & & $\begin{array}{c}\text { APPRL } \\
\text { AMPLAV } \\
\text { DIMUN }\end{array}$ & $\begin{array}{l}-0,330 \\
-0,247 \\
-0,169\end{array}$ & $\begin{array}{l}\text { AMPPTPL } \\
\text { DIMUN }\end{array}$ & $\begin{array}{l}-0,481 \\
-0,328\end{array}$ & AMPLAV & $-0,203$ & ENSSUP & $-0,707$ \\
\hline \multicolumn{11}{|c|}{$\begin{array}{l}\text { REGENERAM } \\
\text { Variável dependente: proporção regenerada no período }\end{array}$} \\
\hline & \multicolumn{2}{|l|}{ ES } & \multicolumn{2}{|c|}{ MG } & \multicolumn{2}{|l|}{$\mathbf{R J}$} & \multicolumn{2}{|l|}{ SP } & \multicolumn{2}{|l|}{ BA } \\
\hline Ajuste melhor Modelo (R) & \multicolumn{2}{|c|}{0,934} & \multicolumn{2}{|c|}{0,707} & \multicolumn{2}{|c|}{0,899} & \multicolumn{2}{|c|}{0,796} & \multicolumn{2}{|c|}{0,980} \\
\hline $\begin{array}{l}\text { (t) Variáveis selecionadas } \\
\text { e coeficientes normalizados }\end{array}$ & APPRL & 0,939 & \begin{tabular}{c|} 
APPRL \\
AMPPTPLA
\end{tabular} & $\begin{array}{l}0,931 \\
0,255\end{array}$ & $\begin{array}{c}\text { APPRL } \\
\text { AMPOPRU }\end{array}$ & $\begin{array}{l}1,073 \\
0,599\end{array}$ & \begin{tabular}{c|} 
APPRL \\
AMPPTPLA
\end{tabular} & $\begin{array}{l}0,814 \\
0,111\end{array}$ & $\begin{array}{c}\text { APPRL } \\
\text { AMPSILV }\end{array}$ & $\begin{array}{l}1,020 \\
0,076\end{array}$ \\
\hline $\begin{array}{l}\text { (-) Variáveis selecionadas } \\
\text { e coeficientes normalizados }\end{array}$ & AMPPTPLA & $-0,167$ & $\begin{array}{c}\text { AREA } \\
\text { AMPSILV }\end{array}$ & $\begin{array}{l}-0,222 \\
-0,169\end{array}$ & & & & & AREA & $-0,136$ \\
\hline
\end{tabular}




\section{DISCUSSÃO}

\section{Regeneração e Desmatamento na Mata Atlântica}

Os dados descritivos mostram que, com exceção do Nordeste, há em todas as regiões mais municípios com regeneração do que com desmatamento líquido de Mata Atlântica. Esse padrão de ressurgimento florestal não é exclusivo desse bioma, tendo sido registrado em vários locais do planeta (ARCE-NAZARIO 2007; BAPTISTA \& RUDEL 2006; RUDEL et al. 2000; MATHER \& NEEDLE 1998). O processo de mudança de uma dinâmica de desmatamento para uma de regeneração de florestas é chamado transição florestal. O modelo atualmente mais utilizado para descrever esse fenômeno é o da EKC - Environmental Kuznets Curve, que postula que a pressão sobre o meio ambiente aumenta, acompanhando o crescimento econômico, até certo limiar, a partir do qual passa a decrescer, havendo então crescimento econômico e recuperação ambiental simultâneos (DINDA 2004). A teoria da transição florestal clássica argumenta que, conforme os países vão se tornando mais urbanizados, áreas rurais vão sendo abandonadas e ocorre a regeneração da vegetação nativa. Tal teoria foi formulada com base na história dos EUA e Europa (HECHT 2008), mas atualmente se reconhece que a transição florestal ocorre por diferentes razões e de modos distintos em cada local (RUDEL et al. 2005). Os resultados da regressão por método stepwise apresentados neste trabalho indicam que, no caso da Mata Atlântica, a transição florestal no bioma está ocorrendo provavelmente em função de uma pressão de atendimento à legislação ambiental, o que é apontado pela consistente e significativa relação entre regeneração e a variável APPRL (proporção de áreas protegidas na forma de APPs e Reservas Legais).

Se os resultados de quantidade de municípios com desmatamento / regeneração demonstram estar havendo um amplo processo de transição florestal no domínio geográfico da Mata Atlântica, por outro lado ainda há desmatamento em termos líquidos no bioma. As localidades em que está havendo desmatamento, notadamente no Mato Grosso do Sul, devem estar desmatando áreas mais extensas do que as áreas que se regeneram nos municípios que podemos chamar "regeneradores". Isso resulta no saldo líquido de decremento das florestas da Mata Atlântica em aproximadamente 2,3 milhões de hectares.

Observa-se ainda que os dados utilizados foram obtidos pelo BGE por meio de entrevistas, em que o produtor rural declara quanto possui de florestas nativas em suas terras. Dessa forma, pode-se especular que os dados de regeneração possam estar superestimadosdevido à popularização da consciência ambiental na última década, o que pode ter levado mais produtores a superdimensionar suas áreas de florestas a fim de responder o que lhe parecia "ecologicamente correto". 


\section{Fatores que influenciam a dinâmica atual do bioma}

Numa análise para o bioma como um todo, observa-se que as principais variáveis relacionadas à dinâmica da Mata Atlântica são AREA (área municipal) e APPRL (proporção de áreas protegidas na forma de APPs e Reservas Legais). Esse padrão quase sempre se repete nas outras escalas, bem como ao se estudar separadamente o subconjunto dos municípios regeneradores e o dos desmatadores. A variável independente AREA (área do município) aparece relacionada positivamente a desmatamento e negativamente a regeneração. Isso demonstra que os municípios maiores estão desmatando mais e recuperando menos florestas, o que pode estar relacionado à capacidade de fiscalização dos órgãos ambientais, que tendem a ter maior dificuldade em vistoriar grandes áreas geográficas. A variável APPRL (proporção de áreas protegidas na forma de APPs e Reservas Legais) também apresenta esse padrão, tendo relação negativa com desmatamento e positivamente com regeneração, o que corrobora a interpretação de que a regeneração da Mata Atlântica está sendo impulsionada pelo "fazer cumprir" as normas da legislação ambiental, especialmente do Código Florestal (Lei 4.771/65 e alterações).

Ampliação de silvicultura (AMPSILV) aparece negativamente relacionada ao desmatamento e positivamente com regeneração. Isso demonstra que municípios com grandes áreas de plantações florestais tendem a apresentar também áreas maiores de vegetação nativa, o que pode estar relacionado a uma prática de conformidade das empresas silvicultoras em relação às obrigações legais, já que esse é um setor onde a conformidade e a certificação ambiental têm um valor considerável.

As ampliações de áreas de pastagens plantadas e lavouras, embora não de forma tão recorrente, também aparecem com relacionadas à diminuição do desmatamento e aumento da regeneração florestal. De maneira semelhante ao analisado para o caso da silvicultura, esses resultados podem indicar uma mudança de comportamento do setor agropecuário nacional na direção do atendimento da legislação ambiental, seja voluntariamente, seja por pressão de fiscalização.

Algumas variáveis, como quantidade de produtores com formação superior e mesmo a ampliação de pastagens plantadas, apresentam um comportamento pouco coerente ao longo dos diversos grupos de municípios. Esse fato, somado à inclusão de alguns fatores apenas para algumas regiões e estados, nos levam a observar que a dinâmica da Mata Atlântica não se comporta de forma homogênea ao longo de todo seu domínio. De fato, trata-se de um bioma de extensões continentais, com grande heterogeneidade de condições naturais, políticas e socioeconômicas em cada porção do território. Essa heterogeneidade é corroborada pelos graus de ajuste apresentados pelos modelos, já que o índice $\mathrm{R}$ do modelo de regressão para o bioma 
completo apresentou-se consideravelmente menor do que os $\mathrm{R}^{\mathrm{ee}} \mathrm{s}$ encontrados para as regiões $\mathrm{e}$ os estados tomados a parte.

Os fatores relacionados à regeneração no Nordeste parecem refletir o mesmo padrão da Bahia, o estado que mais teve aumento florestal em termos líquidos. No estado baiano, a alta regeneração florestal encontrada parece ser fruto de uma forte expansão da silvicultura, acompanhada pela restauração de APPs e Reservas Legais para atendimento à legislação.

Similarmente, o desmatamento no Centro-Oeste apresenta coerência com o padrão encontrado para o Mato Grosso do Sul, que apresenta o maior decremento líquido de Mata Atlântica no período analisado. Não foi detectado nenhum fator que pareça estar estimulando o desmate na região, porém o modelo identificou que os menores desmatamentos ocorrem em municípios com maior quantidade de produtores com formação superior, o que pode ser uma informação útil na elaboração de políticas de combate ao desmatamento do bioma no CentroOeste.

\section{CONCLUSÕES}

Os resultados apresentados neste estudo indicam estar havendo transição florestal na maior parte dos municípios inseridos no domínio da Mata Atlântica. Apesar disso, o saldo global ainda é de decremento do bioma, pois os desmatamentos continuam ocorrendo em grandes extensões. Os resultados da modelagem indicam que a transição florestal que está se iniciando nos municípios é provavelmente motivada por fatores de atendimento à legislação ambiental, como obrigação de manter e restaurar áreas legalmente protegidas nas propriedades rurais (APPs e Reservas Legais).

A diversidade dos modelos obtidos para cada escala e região indica que a Mata Atlântica apresenta padrões sutis e espacialmente diferenciados. Isso não é surpreendente, uma vez que o bioma estende-se do nordeste ao sul do País, atravessando condições físicas, sociais, políticas e econômicas bastante heterogêneas. Ao contrário da Amazônia, cuja expansão de fronteiras agropecuárias é recente e obedece a tendências mais claras, a Mata Atlântica tem um longo e heterogêneo histórico de pressão antrópica, o que resulta numa dinâmica aparentemente mais estocástica, com drivers específicos para cada região do seu domínio. Essa situação diversificada deve ser levada em consideração na formulação de políticas de conservação do bioma, sendo possível que restrições e incentivos específicos para cada região da Mata Atlântica funcionem de maneira mais eficaz que as atuais normas federais generalizantes.

Como é comum para estudos de escalas continentais, é importante reconhecer que os dados utilizados são consideravelmente limitados em termos de precisão, devido à natureza do método usado para sua obtenção. Para que possamos entender a dinâmica da Mata Atlântica e seus drivers, é essencial que os remanescentes do bioma sejam diagnosticados em séries históricas Cadernos do Leste 
mais adequadas à finalidade de monitoramento. Os dados obtidos pelo Censo Agropecuário são oficiais, porém tendem a ser menos objetivos dos que aqueles que poderiam ser obtidos por mapeamentos apropriados. Os mapas atualmente disponíveis para a escala do bioma completo são aqueles fornecidos pelo SOS Mata Atlântica/INPE, cujos métodos de levantamento, continuamente otimizados, limitam a comparação entre os mapas de diferentes períodos. Já há iniciativas estaduais de mapeamentos, como em Minas Gerais e São Paulo, porém seria interessante que a iniciativa se estendesse para todos os estados que se encontram no domínio da Mata Atlântica, com metodologias e critérios equivalentes.

O estudo aqui apresentado está em desenvolvimento e várias perspectivas poderão ser exploradas. Análises do bioma em escalas mais finas e com novas variáveis poderão de grande utilidade para compreensão do objeto de estudo. Podem ser testados modelos de regressão com a inclusão de variáveis que possam melhor representar a influência da atuação de normas e órgãos ambientais, além da expansão das áreas rurais não produtivas (usadas para recreação e residência de temporada, padrão recorrente nas regiões metropolitanas do sudeste). Além disso, modelos de regressão espacial permitirão avaliar qual a influência da vizinhança sobre os padrões de desmatamento e regeneração do bioma. Todas essas análises permitirão uma melhor compreensão da dinâmica da Mata Atlântica e do processo detransição florestal observado neste trabalho, podendo contribuir para a definição de estratégias de conservação mais adequadas ás diversas facetas do bioma.

\section{REFERÊNCIAS BIBLIOGRÁFICAS}

AGUIAR, A.P.D., CAMARA, G. \& ESCADA, M.I.S. Spatialstatisticalanalysisofland-use determinants in theBrazilianAmazonia: exploringintra-regionalheterogeneity, EcologicalModelling, v. 209, n.2-4, p.169-188, 2007.

ANDERSEN, L.E. Modelling the Relationship between Government Policy, Economic Growth, and Deforestation in the Brazilian Amazon. Disponível em SSRN: http://ssrn.com/abstract=54986 ordoi:10.2139/ssrn.54986. Acessoem: 24 nov. 2010.

ARCE-NAZARIO, J.A. Human landscapes have complex trajectories: reconstructing Peruvian Amazon landscape history from 1948 to 2005. Landscape Ecology, v. 22, n. S1, p.89-101, 2007.

BAPTISTA, S.R. \& RUDEL, T. K. A re-emerging Atlantic forest? Urbanization, industrialization and the forest transition in Santa Catarina, southern Brazil. Environmental Conservation, v. 33, n.03, p.195-202, 2006.

DINDA, S. Environmental Kuznets Curve Hypothesis: a survey. EcologicalEconomics, v. 49, n.4, p.431-455, 2004. 
EHLERS, E.M. Determinantes da Recuperação da Mata Atlântica no Estado de São Paulo. 2003. 351f. Tese de Doutorado (Pós-Graduação em Ciências Ambientais). Programa d Pós-Graduação em Ciências Ambientais - PROCAM, USP, São Paulo, 2003.

GARCIA, R. A.; LEMOS, M. B.. A migração como variável endógena: caracterização do processo de transformação das regiões de influência dos pólos econômicos brasileiros. Economia, v. 10, n. 2, p. 253-275, 2009.

HECHT, S. The New Rurality: globalization, peasants and the paradoxes of landscapes. Desenvolvimento e Meio Ambiente, v.17, p.141-160, 2008.

LORENA, R.B. \& LAMBIN, E.F..The spatial dynamics of deforestation and agent use in the Amazon. Applied Geography, v. 29, n. 2, p.171-181, 2009.

MATHER, A S. \& NEEDLE, C.L. The forest transition: a theoretical basis. Area, v.30, n.2, p.117-124, 1998.

MICHALSKI, F., METZGER, J.P. \& PERES, C. A. Rural property size drives patterns of upland and riparian forest retention in a tropical deforestation frontier. Global Environmental Change, v.20, n.4, p.705-712, 2010.

PERZ, S.G. \& SKOLE, D.L. Secondary Forest Expansion in the Brazilian Amazon and the Refinement of Forest Transition Theory. Society \& Natural Resources, v.16, n.4, p.277-294, 2003.

REIS, E.J., BLANCO, F.A. \& DIPES, I. The Causes of Brazilian Amazon Deforestation. Preliminary Draft of the wider project The Forest in the South and the North in the Context of Global Warming, Amazon Sub-Study. IPEA, Rio de Janeiro, Fev. 1997. Disponível em: http://www.nemesis.org.br/sec-din.php?s=500\&i=pt. Acesso em: 24 nov. 2010.

RIBEIRO, M.C., METZGER, J.P., MARTENSEN, A.C., PONZONI, F.J. \& HIROTA, M.M. The Brazilian Atlantic Forest: How much is left, and how is the remaining forest distributed? Implications for conservation. Biological Conservation, v.142, n.6, p.1141-1153, 2009.

RUDEL, T.K., COOMES, O.T., MORAN, E., ACHARD, F., ANGELSEN, A., XU, JIANCHU \& LAMBIN, E. Forest transitions: towards a global understanding of land use change. Global Environmental Change, v.15, p.23-31, 2005.

RUDEL, T.K, PEREZ-LUGO, M. \& ZICHAL, H. When Fields Revert to Forest: Development and Spontaneous Reforestation in Post-War Puerto Rico. Professional Geographer, v.52, n.3, p.386-397, 2000.

SILVA, J.H.G.D. Economic Causes of Deforestation in the Brazilian Amazon : An Empirical Analysis of the 2000s. Disponivel em: http://www.nemesis.org.br/sec-din.php?s=500\&i=pt. Acesso em: 24 nov. 2010.

SOARES-FILHO, B.S., GACIA, R.A., RODRIGUES, H.O., MORO, S. \& NEPSTAD, D.C. Nexos entre as dimensões socioeconômicas e o desmatamento da Amazônia: a caminho de um modelo integrado. In: BATISTELLA, M.; ALVES, D.; MORAN, E. (Ed.). Amazônia: Natureza e Sociedade em Transformação. São Paulo: Edusp, 2008. P. 181-217.

TEIXEIRA, A.M.G., SOARES-FILHO, B.S., FREITAS, S.R., METZGER, J.P. Modelinglandscape dynamics in anAtlanticRainforestregion: Implications for conservation. Forest Ecologyand Management, v.257, n.4, p.1219-1230, 2009.

Cadernos do Leste

Artigos Científicos

Belo Horizonte, Dez. Vol.10, n¹0, 2010 
WU, J. \& HOBBS, R., 2002. Key issues and research priorities in landscape ecology : An idiosyncratic synthesis. LandscapeEcology, v.17, p.355-365, 2002. 